auch im Leben strebt man oftmals das Maximale an, um danach das real Erreichte als optimal zu deklarieren. Und so kam alles ganz anders...

Nach der Zusammenlegung der Institute für Niederfrequenztechnik und Hochfrequenztechnik gab es mehrere Jahre, nun sagen wir, heftigen Wettbewerb darum, welcher Arbeitsstil bevorzugt werden sollte.

Mit großer Umsicht, Geduld und Zähigkeit hat HW während seiner Tätigkeit als Institutsvorstand eingefahrene Strukturen am Institut bereinigt und neu aufgebaut; auch wenn seine Zeit als Institutsvorstand nur drei Jahre dauerte. Das Spektrum der wissenschaftlichen Themen wurde gestrafft und das gemeinsame Institut viel homogener. Ich ging auch wieder viel lieber zur täglichen Arbeit am Institut.

HW ist sehr ausdauernd. Seine Zähigkeit haben wir alle, die in der guten alten Zeit mittäglich Tischtennis spielten, kennen und fürchten gelernt. Man konnte noch so hart auf den Ball „dreschen", noch so gefinkelt schneiden, Hans hat alle Bälle zurückgebracht (obwohl man doch in Wien sprichwörtlich sagt: eine Reaktion haben wie ein Schachspieler). Hans, auch ein Schachspieler, hat uns zur Verzweiflung gebracht!

Wenn man Hans mit einer wissenschaftichen Frage konfrontiert, geht man nie ungetröstet aus seinem Zimmer. Seine Fähigkeit, das, was man vielleicht gar nicht so genau formulieren kann, als Frage zu erkennen und darauf eine Antwort zu geben, die das Niveau des Fragenden haargenau trifft, $d . h$. dass man seine Antwort sofort verwenden kann, das habe ich in meiner Laufbahn nur bei ihm erlebt. Und bei Hans wagt man es auch, mit scheinbar trivialen Fragen aufzukreuzen, weil er einem nie das Gefühl vermittelt: $\mathrm{Na}$, das hättest du doch selbst wissen müssen.

In der Führung seiner Mitarbeiter beweist Hans immer wieder außerordentliches Geschick. Er spürt ihre verborgenen Talente auf und führt sie zu immens wertvollen Dissertationen und
Veröffentlichungen. Wir haben ihm - zu unserer Schande sei es gesagt - viel zu wenig Mitarbeiter gegönnt.

Etwa 1997 wurde ich um die Begutachtung eines Manuskripts über Mehrantennensysteme ersucht. Die Autoren versprachen wunderliche Dinge: spektrale Effizienzen von einigen zehn bit/s pro Hertz Bandbreite. Nach allem, was

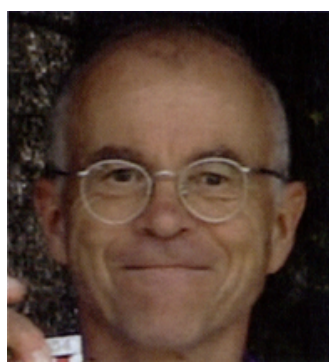
mir von Informationstheorie hängen geblieben war und was ich von Antennengruppen zu wissen glaubte, konnte es das einfach nicht geben. Schließlich beeinflussten sich Antennen in unmittelbarer Nähe voneinander stark und in recht undurchsichtiger Weise. Und Experimente waren zur Stützung der Behauptungen ebenfalls nicht angeführt. Man kann sich meine Skepsis vorstellen. Andererseits: offensichtliche Fehler waren in den Annahmen, Berechnungen und Argumenten nicht zu finden. Was tun? Also fragt ich Hans um einen vertraulichen Rat. Er sah sich das Manuskript an, konnte keinen gravierenden Fehler finden und riet mir, einer Veröffentlichung zuzustimmen. Gestärkt durch Hansens Meinung gab ich, mit einem flauen Gefühl im Magen allerdings, meinen Widerstand gegen die Veröffentlichung des Manuskripts auf. Fußnote: Heute ist dieser Artikel einer der am häufigsten zitierte bei MIMO-Veröffentlichungen.

Ärgerlich kann Hans nur werden, wenn man Code oder Codierung - sein Lieblingsfach - mit $\mathrm{K}$ schreibt. Aber wir werden tunlichst vermeiden, inn zu ärgern. Ganz im Gegenteil; wir wünschen Hans noch viele zufriedene Jahre bei vollster Schaffenskraft!

O. Univ.-Prof. i. R. Dipl.-Ing. Dr. techn. Ernst Bonek Institut für Nachrichtentechnik und Hochfrequenztechnik Technische Universität Wien

\title{
Nachrichtentechnik - von Filterung bis Codierung
}

\section{Liebe Leserin, lieber Leser,}

die wissenschaftlichen Beiträge der vorliegenden e \& i-Ausgabe sind Herrn Prof. Hans Weinrichter zu seinem 65. Geburtstag gewidmet. Die große Spannweite der Themen, die Professor Weinrichter in seinem Berufsleben bearbeitet und zu denen er wesentliche Beiträge geleistet hat, kann durch die Beiträge allerdings nicht abgedeckt werden. Dazu würde auch ein einzelnes Heft nicht ausreichend Platz bieten.

So ist es selbstverständlich, dass die hier zusammengefassten Beiträge die aktuellen Arbeitsgebiete von Professor Weinrichter berühren. Dabei geht es um Signalverarbeitung und Codierung für die Mobilkommunikation. Was macht eigentlich die Mobilkommunikation so attraktiv, was hat zu dieser enormen Verbreitung, die wir alle erleben und zu der wir beigetragen haben, geführt? Trotz aller Schwierigkeiten, der beschränkt ver- fügbaren Bandbreite, der großen Variabilität des Mobilfunkkanals über Zeit, Frequenz und Raum ist die Kommunikation mit Hilfe mobiler Endgeräte auf ihrem Vormarsch nicht zu bremsen.

Um die Herausforderungen neuer Dienste, die vielfach hohe Datenraten bedingen, meistern zu können, sind neue Konzepte, ja neue Dimensionen notwendig. Es genügt nicht mehr, die informationstragenden Signale in der Zeit zu verarbeiten, wir müssen die Signale auch als Funktion des Raumes begreifen und mit Hilfe der Raum-Zeit-Signalverarbeitung ein neues Kapitel der Übertragungstechnik, der Signalverarbeitung und Codierung aufschlagen. Systeme mit mehreren Antennen an beiden Enden der Funkstrecke, so genannte MIMO(Multiple-Input-Multiple-Output)-Systeme bieten die Möglichkeit zur dramatischen Steigerung der Übertragungskapazität bei gegebener $\mathrm{Be}-$ schränkung der Bandbreite und Sendeleistung. 
Die ersten vier Beiträge in dieser Ausgabe stellen unterschiedliche Aspekte der aktuellen Forschung auf dem Gebiet der MIMO-Systeme dar:

Der Beitrag von R. Fischer und J. Huber gibt einen Überblick über die Entzerrungsverfahren, die von Ein-Antennensystemen hier für MIMO-Systeme weiterentwickelt wurden. Es werden sowohl lineare als auch nichtlineare Verfahren zur Anwendung im Empfänger und auch im Sender (Vorverzerrung, Precoding) dargestellt, ebenso wird der Blick auf derzeit noch ungelöste Fragen gelenkt.

Um die komplexen Signalverarbeitungsverfahren zu bewerten, sind Modelle für MIMO-Kanäle erforderlich. Diesem Thema sind die nächsten zwei Beiträge von $\mathrm{E}$. Bonek bzw. von $\mathrm{B}$. Lankl, A. Knopp und M. Chouayakh gewidmet. In der Arbeit von $E$. Bonek werden in der aktuellen Forschung häufig verwendete Kanalmodelle anhand von Messergebnissen kritisch verglichen und bewertet. Man erhält wichtige Hinweise, für welche Szenarien welche Kanalmodelle geeignet sind. Trotz zahlreicher interessanter Ergebnisse ist auch auf diesem Gebiet noch viel zu tun, um das theoretische Potential von MIMO-Systemen praktisch ausschöpfen zu können.

In der Arbeit von Lankl et al. werden ebenfalls Kanalmessungen beschrieben und ausgewertet, hier mit dem Schwerpunkt auf Indoor-MIMO-WLAN-Anwendungen. Die Auswertun-

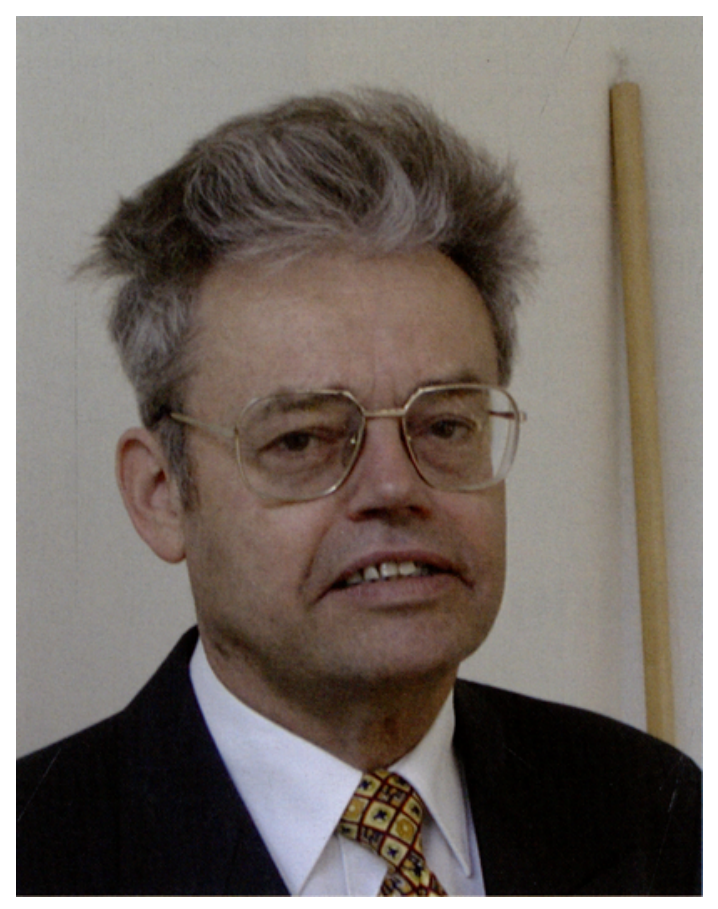

Univ.-Prof. Dr. Hans Weinrichter vollendete im März sein 65. Lebensjahr gen geben wichtige Hinweise für die Antennenanordnungen im Hinblick auf maximale Übertragungskapazität.

Der vierte Beitrag von $\mathrm{J}$. Nossek und M. Ivrlač versucht, Kennzahlen für Antennengewinn, Diversitätsgewinn und Multiplexgewinn von MIMO-Systemen anzugeben, mit deren Hilfe Signalverarbeitungsverfahren bewertet bzw. optimiert werden können. Eine dieser Kennzahlen wird bereits in der Arbeit von E. Bonek beim Vergleich verschiedener Kanalmodelle benutzt.

Der fünfte und letzte Beitrag von J. Hagenauer wendet sich einem anderen Thema $z u$, nämlich der Datenschätzung von über AWGN- bzW. Schwundkanäle übertragenen binären Sendesymbolen mit ungleicher Wahrscheinlichkeit.

Alle Beiträge haben eine große Nähe zu den neueren Arbeiten von Professor Weinrichter. Ich möchte hier noch den Bogen spannen von den Anfängen, die ich aus eigener Erfahrung kenne, bis hin zu den aktuellen Beiträgen dieses Heftes. Ich habe Herrn Weinrichter während meines Studiums an der TU Wien kennen gelernt. Er hat meine Diplomarbeit auf dem Gebiet der RC-aktiven Filter betreut. Während eines Jahres, das Professor Weinrichter als Gastwissenschaftler bei der Siemens AG in München im damaligen Zentrallaboratorium für Nachrichtentechnik verbrachte, begann er meine Doktorarbeit auf dem Gebiet der Schalter-Kondensator-Filter, einem damals hoch aktuellen Thema, zu betreuen.

Wieder einige Jahre später konnte ich Herrn Weinrichter als Berater gewinnen, der mich bei der Einführung der Digitalsignalübertragung im Richtfunk wesentlich unterstützte. Die Themen haben also mit klassischer Filterung begonnen und sich zu Signalverarbeitung und Codierung in Kommunikationssystemen hin entwickelt. Aber sind nicht adaptive Entzerrer und sendeseitige Vorcodierer auch Filter? So habe ich über weite Abschnitte meines Berufslebens vom Gedankenaustausch mit Prof. Weinrichter und von seinem Rat wesentlich profitiert. Ich bin für diese Zusammenarbeit sehr dankbar und möchte mit diesem Heft meine Dankbarkeit und Anerkennung und die meiner Kollegen, die mit ihren Beiträgen dieses Heft möglich gemacht haben, ausdrücken.

Ich wünsche Herrn Prof. Weinrichter viel Freude an den ihm gewidmeten Beiträgen und noch viele zufriedene Jahre bei guter Gesundheit im aktiven Ruhestand.

Univ.-Prof. Dr. Josef A. Nossek

Institut für Netzwerktheorie und Signalverarbeitung

Technische Universität München 